\title{
Colitis microscópica. Un diagnóstico a tener en cuenta en caso de diarrea secretora
}

\author{
M. J. MENDUIÑA GUILLÉN, P. ALAMINOS GARCÍA, M. VALENZUELA \\ BARRANCO
}

Departamento de Medicina. Universidad de Granada

\section{RESUMEN}

La colitis microscópica se caracteriza por diarrea acuosa crónica acompañada o no de dolor abdominal. Se presenta en pacientes de mediana edad y el diagnóstico se realiza mediante los hallazgos microscópicos de una mucosa de colon con un aspecto macroscópico normal. Existen 2 tipos microscópicamente distintos, la colitis colágena y la colitis linfocítica.

Presentamos 2 casos de cada uno de los tipos de colitis microscópica, cuya manifestación clínica en común era la diarrea secretora crónica.

PALABRAS CLAVE: Colitis microscópica. Colitis colágena. Colitis linfocítica.

MICROSCOPIC COLITIS. A DIAGNOSIS TO HAVE TO DEBIT IN CASE OF WATERY DIARRHEA

Menduiña Guillén MJ, Alaminos García P, Valenzuela Barranco M. Colitis microscópica. Un diagnóstico a tener en cuenta en caso de diarrea secretora. An Med Interna (Madrid) 2004; 21: 387-390.

\section{INTRODUCCIÓN}

La colitis microscópica fue descrita por primera vez en 1976 (1). Se caracteriza clínicamente por diarrea secretora (acuosa) crónica, acompañada o no de dolor abdominal y de otros síntomas como vómitos y urgencia fecal $(2,6)$; normalmente se presenta en pacientes de mediana edad (50-70 años). Su principal característica es que, el diagnóstico habitualmente se realiza mediante los hallazgos microscópicos de una mucosa de colon con un aspecto macroscópico normal.

Existen dos tipos, según dichos hallazgos microscópicos:

1. Colitis colágena: de mayor prevalencia en mujeres (8) (en algunas series se establecen proporciones de 9 mujeres por cada varón), caracterizada por un infiltrado inflamatorio en la lámina propia con la existencia de depósitos subepiteliales de colágeno de 7-100 $\mu$ de grosor (3).

2. Colitis linfocítica: de igual prevalencia en mujeres que en hombres (8), cuyos hallazgos histológicos característicos son la presencia de infiltrado inflamatorio a base de células mononucleares con ausencia de neutrófilos en la lámina pro-

\section{ABSTRACT}

The colitis microscopic was characterized by chronic watery diarrhea accompanied or not of abdominal pain. Present in patients of median age and the diagnosis was realized by means of the microscopic finds of a mucus of colon with an aspect macroscopic normal. They exist 2 types microscopically distinct, the collagenous colitis and the lymphocytic colitis.

We referred 2 cases of each one of the types of microscopic colitis, whose manifestation clinic in common was the chronic watery diarrhea.

KEY WORDS: Microscopic colitis. Collagenous colitis. Lymphocytic colitis. 
nal vasoactivo (VIP): $5 \mathrm{pg} / \mathrm{ml}$ (valores normales: 0-70 pg/ml). Niveles de ácido 5-hidroxindol-acético (5HIAA) en orina de 24 horas: 0,5 $\mathrm{mg} / 24$ horas (las cifras normales son inferiores a $10 \mathrm{mg} / 24$ horas). PCR: $1,2 \mathrm{mg} / \mathrm{dl}$ (cifras normales en nuestro laboratorio hasta 0,8 $\mathrm{mg} / \mathrm{dl}$ ), VSG: $46 \mathrm{~mm}$ en $1^{\mathrm{a}}$ hora. Anticuerpos antiendomisio y antigliadina negativos Leucocitos en heces: negativos. Coprocultivos, e investigación de $\mathrm{Cl}$ difficile, de parásitos y de huevos de estos en heces: negativos. Tránsito esófago-gastro-duodenal (TEGD): normal. Ecografía abdominal: normal salvo aerobilia con ausencia de vesícula biliar. Colonoscopia e ileoscopia: sin alteraciones macroscópicas. Se toman 6 biopsias a lo largo de todo el colon (incluido sigma y recto), observándose en todas ellas un importante infiltrado inflamatorio con gran cantidad de linfocitos intraepiteliales (Fig. 1).

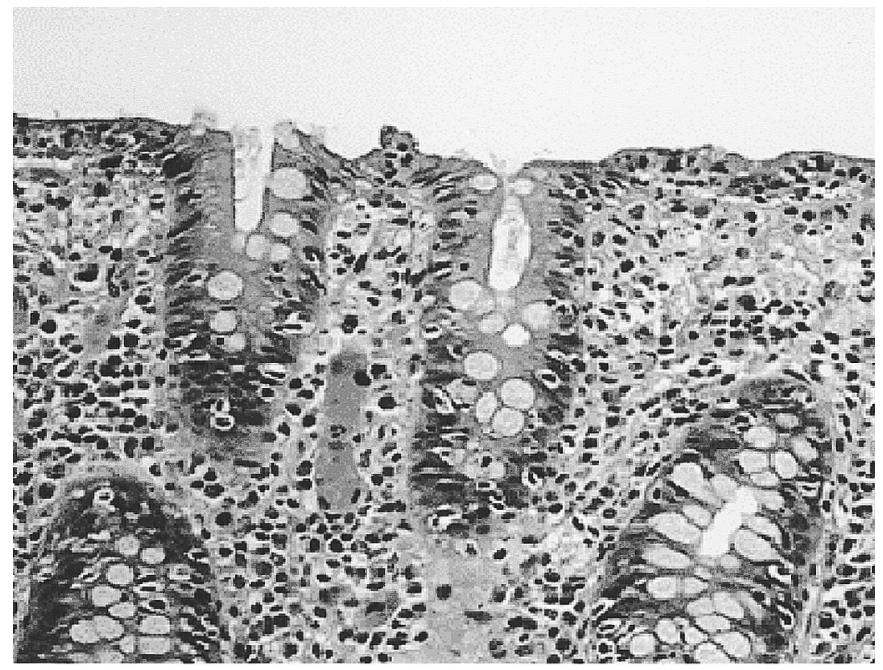

Fig. 1.

Caso 2: varón de 62 años que presenta como único antecedente personal, una lumbalgia crónica, que trata habitualmente con naproxeno. Acude a consulta por diarrea acuosa sin fiebre, acompañada de sensación de tenesmo rectal, de casi dos meses de evolución. Exploración física: sin ningún hallazgo significativo. Hematimetría, coagulación y bioquímica con ionograma bilirrubina y fosfatasa alcalina con cifras dentro de los valores de normalidad. PCR: 0,95 mg/dl, VSG: 60 en al $1^{a}$ hora. TSH y hormonas tiroideas: con cifras normales. Niveles de ácido 5-hidroxindol-acético (5HIAA) en orina de 24 horas: 2 $\mathrm{mg} / 24$ horas (dentro de la normalidad). Niveles plasmáticos de péptido intestinal vasoactivo (VIP): $1 \mathrm{pg} / \mathrm{ml}$. Anticuerpos antigliadina y antiendomisio negativos. Leucocitos en heces: negativos. Coprocultivos seriados e investigación de $\mathrm{Cl}$ difficile, de parásitos y de huevos de estos en heces: negativos. Tránsito esófagogastroduodenal. (TEGD) y ecografía abdominal: sin alteraciones. Colonoscopia e ileoscopia: aspecto macroscópico de la mucosa normal sin otras alteraciones. Se toman 6 biopsias en colon, sigma y recto. En todas ellas se observan abundantes engrosamientos subepiteliales de colágeno de al menos $100 \mu$ micras, además de un abundante infiltrado inflamatorio en el que predominan las células mononucleares (Fig. 2).

En el primer caso, el patólogo nos sugiere el diagnostico de una colitis linfocítica, y en el segundo de una colitis colágena. En ambos casos, dada la escasa respuesta a loperamida y restricción de AINE, se prescribe tratamiento con dieta baja en residuos y mesalazina a dosis de $2.400 \mathrm{mg} /$ día durante ocho semanas, tras las cuales desaparece la diarrea secretora, permaneciendo ambos pacientes asintomáticos a los ocho meses y un año respectivamente de finalizar el tratamiento. En ambos casos se propuso una nueva exploración endoscópica con el objetivo de comprobar la remisión histológica de la enfermedad, pero los pacientes rechazaron dicha posibilidad.

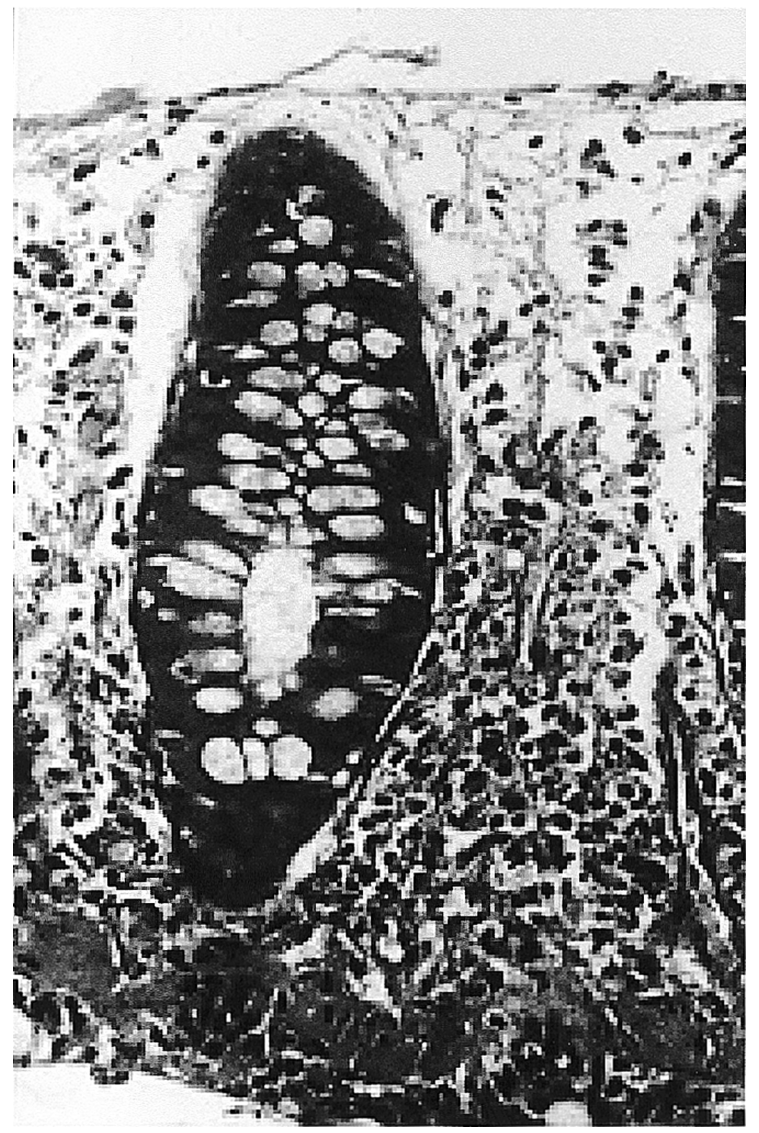

Fig. 2.

\section{DISCUSIÓN}

La colitis microscópica (en sus dos formas) es un diagnóstico a tener en cuenta en todo paciente de mediana edad con diarrea secretora de curso crónico, siempre que se hayan descartado previamente otras causas como: diarreas infecciosas, diarrea facticia, VIPOMA, cólera, síndrome carcinoide e hipertiroidismo (9). En cuanto a la etiología, los distintos autores han querido implicar, factores autoinmunitarios (11), toxinas bacterianas (como la toxina del $\mathrm{Cl}$ difficile) $(12,13)$ y el consumo de algunos fármacos, tales como lansoprazol, simvastatina, ticlopidina, flutamida (14-16) y los antinflamatorios no esteroideos (AINES). Referidos a éstos últimos se ha encontrado alguna evidencia de asociación entre su consumo y la incidencia de colitis colágena (17); si bien es cierto que otros trabajos más recientes parecen descartar esta asociación $(18,19)$. Por otra parte en los últimos años se ha sugerido una relación etiológica entre la colitis microscópica y la enfermedad celíaca, debido a la concurrencia descrita de esta última patología sobre todo con la colitis linfocítica (20). Algunos autores relacionan también a la colitis microscópica con la enfermedad inflamatoria intestinal por diversas lesiones histológicas propias de la primera que pueden aparecer tanto en la colitis ulcerosa como en la enfermedad de Crohn, sugiriendo incluso que los cambios histopatológicos característicos de la colitis microscópica pueden preceder al desarrollo tanto clínico como anatomopatológico de la enfermedad inflamatoria intestinal; particularmente de la enfermedad de Crohn $(21,22)$. 
Referente al diagnóstico: puede existir aumento de los parámetros de fase aguda (PCR y VSG), así como leucocitos en las heces en casi la mitad de los casos (23); lo cual confirma la base inflamatoria de la enfermedad. Pero el diagnóstico de certeza nos lo dan los hallazgos microscópicos obtenidos mediante biopsia múltiple (al menos 6) incluyendo, recto e ileon si es posible, de una mucosa de colon con aspecto macroscópico normal. Puede entenderse por tanto, la importancia de la colonoscopia con toma de biopsias múltiple, para el diagnóstico de dicha enfermedad (24).

En cuanto al tratamiento no existe un estándar consensuado, puesto que hay casos en los que puede haber una remisión tras la suspensión de tratamientos previos con AINE (19); incluso se han descrito remisiones espontáneas hasta en un tercio de los casos $(25,26)$. El tratamiento sintomático con loperamida, opiáceos y difenoxilato, produce resultados variables en el control de la diarrea, según los distintos autores (24). También se han comunicado remisiones clínicas con colestiramina, reivindicando así un hipotético papel de los ácidos biliares, cuya malabsorción podría estar implicada en la patogenia de la enfermedad (27). Una buena opción terapéutica son los corticoides, sobre todo la budesonida por vía oral con la cual, en un estudio reciente, (con dosis de $9 \mathrm{mg}$ al día durante 6 semanas), se consiguió una rápida remisión tanto clínica como histológica en pacientes con colitis colágena, minimizando los efectos secundarios achacados a otros corticoides (28). Los aminosalicilatos como la sulfasalazina (29), (a dosis de 2-3 g al día) olsalazina (30) y sobre todo la mesalazina (31) (a dosis de 1,2-4 g al día) se han usado también con gran éxito; lo cual viene a reforzar la referida similitud de esta entidad clínica con la enfermedad inflamatoria intestinal. Otras opciones terapéuticas, han demostrado su efectividad en series cortas, refractarias al tratamiento con aminosalicilatos y corticoides; entre ellas se incluyen: metronidazol (32) y en casos de diarrea severa, methotrexate, azathioprina, 6-mercaptopurina (33) y octeótrido subcutáneo (34). Solamente en casos de colitis microscópica refractaria al tratamiento médico y con gravedad manifiesta, es necesario emplear la cirugía y concretamente la ileostomía temporal; intervención que en la mayoría de casos no es definitiva, dado que suele producirse una recaída al restablecer la continuidad del tubo digestivo (35).

En cuanto a la respuesta al tratamiento, se ha sugerido que la duración del periodo sintomático previo al inicio del tratamiento, puede influir en la respuesta a este, siendo en general pobre cuando dicho periodo supera los tres meses $(26,36)$. Recientemente Abdo y cols. han publicado un trabajo en el que partiendo de un grupo de pacientes con colitis colágena, se concluye que el grado de inflamación existente a nivel de la lámina propia es un factor predictor de la respuesta al tratamiento, sirviéndonos este dato de guía para elegir el fármaco adecuado. De esta forma parece que los pacientes que tienen un grado de inflamación importante de la lámina propia, precisarán al menos, corticoides para obtener remisión, mientras que aquellos que muestran poca o mínima inflamación responderán a antidiarreicos habituales, a la 5-ASA o incluso, remitirán de manera espontánea. En este trabajo se concluyó también que los pacientes con remisión espontánea o que responden a antidiarreicos, tienen significativamente una mayor edad y que aquellos que consumen AINE, suelen precisar corticoides para el control de los síntomas, por desarrollar formas más severas de enfermedad (37).

Como pauta terapéutica se aconseja comenzar por el tratamiento sintomático; si fracasa emplear aminosalicilatos (sulfasalazina, mesalazina) y sólo cuando estos fracasan usar los corticoides sistémicos (26), principalmente budesonida vía oral.

La respuesta al tratamiento varía desde la remisión completa clínica e histológica hasta la mejoría sintomática con persistencia de las alteraciones histológicas. Un curso clínico con una evolución crónica intermitente puede obligar a ciclos repetidos de tratamiento $(38)$.

Para finalizar diremos que hay autores que no están de acuerdo con considerar colitis colágena y colitis linfocítica como dos formas de una misma enfermedad, considerando a aquellas como dos entidades totalmente independientes (39). De cualquier forma, habiendo revisado la literatura de manera exhaustiva, todo parece indicar que no existen diferencias significativas en cuanto al diagnóstico tratamiento y curso evolutivo de estas dos entidades clínicas (40), independientes o no.

\section{Bibliografía}

1. Lindstrom CG. Collagenous colitis' with watery diarrhoea-a new entity? Pathol Eur 1976; 11 (1): 87-9.

2. Sylwestrowicz T, Kelly JK, Hwang WS, Shaffer EA. Collagenous colitis and microscopic colitis: the watery diarrhea-colitis syndrome. Am J Gastroenterol 1989; 84 (7): 763-8.

3. Lee E; Schiller LR; Vendrell D; Santa Ana CA; Fordtran JS. Subepithelial collagen table thickness in colon specimens from patients with microscopic colitis and collagenous colitis Gastroenterology 1992; 103 (6): 1790-6.

4. Giardiello FM, Lazenby AJ; Bayless TM, Levine EJ, Bias WB, Ladenson PW, Hutcheon DF, Derevjanik NL, Yardley JH. Lymphocytic (microscopic) colitis. Clinicopathologic study of 18 patients and comparison to collagenous colitisDig Dis Sci 1989; 34 (11): 1730-8.

5. Mills LR; Schuman BM; Thompson WO. Lymphocytic colitis. A definable clinical and histological diagnosis..Dig Dis Sci 1993; 38 (6): 1147-51.

6. Pardi DS, Smyrk TC, Tremaine WJ, Sandborn WJ. Microscopic colitis: a review. Am J Gastroenterol 2002; 97 (4): 794-802.

7. Ayata G, Ithamukkala S, Sapp H, Shaz BH, Brien TP, Wang HH, Anto-

nioli DA, Farraye FA, Odze RD. Prevalence and significance of inflammatory bowel disease-like morphologic features in collagenous and lymphocytic colitis. Am J Surg Pathol 2002; 26 (11): 1414: -23.

8. Fernández-Banares F, Salas A, Forne M, Esteve M, Espinos J, Viver JM. Incidence of collagenous and lymphocytic colitis: a 5-year population- based study. Am J Gastroenterol 1999; 94 (2): 418-23.

9. Donowitz M; Kokke FT; Saidi R. Evaluation of patients with chronic diarrhea. N Engl J Med 1995; 332 (11): 725-9.

10. Lewis FW, Warren GH, Goff JS. Collagenous colitis with involvement of terminal ileum. Dig Dis Sci 1991; 36 (8): 1161-3.

11. Germany RE, Cohen SM. Hepatitis C, collagenous colitis, and dermatomyositis occurring in the same patient. Am J Gastroenterol 2002; 97 (7): 1848-9.

12. Andersen T, Andersen JR, Tvede M, Franzmann MB. Collagenous colitis: are bacterial cytotoxins responsible? Am J Gastroenterol 1993; 88 (3): 375-7.

13. Khan MA, Brunt EM, Longo WE, Presti ME. Persistent clostridium difficile colitis: a possible etiology for the development of collagenous colitis. Dig Dis Sci 2000; 45 (5): 998-1001. 
14. Wilcox GM, Mattia A. Collagenous colitis associated with lansoprazole. J Clin Gastroenterol 2002; 34 (2): 164-6.

15. Baert F, Wouters K, D'Haens G, Hoang P, Naegels S, D'Heygere F, Holvoet J, Louis E, Devos M, Geboes K. Lymphocytic colitis: a distinct clinical entity? A clinicopathological confrontation of lymphocytic and collagenous colitis. Gut 1999; 45 (3): 375-81.

16. Feurle GE, Bartz KO, Schmitt-Graff A. Lymphocytic colitis, induced by ticlopidine. Z Gastroenterol 1999; 37 (11): 1105-8.

17. Riddell RH, Tanaka M, Mazzoleni G. Non-steroidal anti-inflammatory drugs as a possible cause of collagenous colitis: a case-control study. Gut 1992; 33 (5): 683-6.

18. Veress B, Lofberg R, Bergman L. Microscopic colitis syndrome. Microscopic colitis syndrome. Gut 1995; 36 (6): 880-6.

19. Beaugerie L, Berenbaum F, Berrebi D, Gendre JP, Prier A, Kaplan G, Chatelet FP. Chronic use of non-steroidal anti-inflammatory drugs does not alter colonic mucosa of patients without diarrhoea.. Aliment Pharmacol Ther 2001; 15 (9): 1301-6.

20. Gillet, HR, Freeman, HJ. Prevalence of celiac disease in collagenous and lymphocytic colitis. Can J Gastroenterol 2000; 14: 919.

21. Goldstein NS, Gyorfi T. Focal lymphocytic colitis and collagenous colitis: patterns of Crohn's colitis? Am J Surg Pathol 1999 Sep; 23 (9): 1075-81.

22. Goldblum JR, Wang N. Lymphocitic colitis and collagenous collitis as possible patterns of Crohn's colitis (letter). Am J Surg Pathol 2000; 24: 775.

23. Zins BJ, Tremaine WJ, Carpenter HA. Collagenous colitis: mucosal biopsies and association with fecal leukocytes. Mayo Clin Proc 1995; 70 (5): 430-3.

24. Fine KD Seidel RH Do K. The prevalence, anatomic distribution, and diagnosis of colonic causes of chronic diarrhea [In Process Citation]. Gastrointest Endosc 2000; 51 (3): 318-26.

25. Teglbjaer PS, Thaysen EH, Jansen. Development of collagenous colitis in sequential biopsy specimens. Gastrenterology 1984; 87: 703-709.

26. Díaz Blasco J, García Valriberas R. Colitis colágena y linfocítica. Med Clin (Barc) 1997; 108: 791-796.

27. Ung KA, Gillberg R, Kilander A, Abrahamsson H. Role of bile acids and bile acid binding agents in patients with collagenous colitis. Gut
2000; 46 (2): 170-5.

28. Miehlke S, Heymer P, Bethke B, Bastlein E, Meier E, Bartram HP et al. Budesonide treatment for collagenous colitis: a randomized, doubleblind, placebo-controlled, multicenter trial. Gastroenterology 2002; 123 (4): $978-84$

29. Weidner N, Smith J, Pattee B. Sulfasalazine in treatment of collagenous colitis. Case report and review of the literature Am J Med 1984; 77 (1): $162-6$.

30. Alberti-Flor JJ, Howell S. Olsalazine in collagenous colitis. Br J Clin Pract 1994; 48 (3): 158-9.

31. Katanuma A, Kodama T, Tamaki T, Katabami S, Yamashita K, Itoh J, et al. Collagenous colitis. Intern Med 1995; 34 (3): 195-8.

32. Mogensen AM, Olsen JH, Gudman-Hoyer E. Collagenous collitis. Acta Med Scand 1984; 216: 535-540.

33. Pardi DS, Loftus EV Jr, Tremaine WJ, Sandborn WJ. Treatment of refractory microscopic colitis with azathioprine and 6-mercaptopurine. Gastroenterology 2001; 120 (6): 1483-4.

34. Fisher NC, Tutt A, Sim E, Scarpello JH, Green JR. Collagenous colitis responsive to octreotide therapy. J Clin Gastroenterol 1996; 23 (4): 3001 .

35. Jarnerot G, Tysk C, Bohr J, Eriksson S. Collagenous colitis and fecal stream diversion. Gastroenterology 1995; 109 (2): 449-55

36. Rams H, Rogers AI, Ghandur-Mnaymneh L. Collagenous collitis Ann Intern Med 1987; 106: 108-113.

37. Abdo A, Raboud J, Freeman HJ, Zetler P, Tilley J, Chaun H, et al.Clinical and histological predictors of response to medical therapy in collagenous colitis. Am J Gastroenterol 2002; 97 (5): 1164-8.

38. Naranjo Rodríguez A, Mino Fugarolas G. Diagnostic criteria and therapeutic management of collagenous colitis. Rev Clin Esp 2001; 201 (10): 594-5.

39. Lee E, Schiller LR, Vendrell D, Santa Ana CA, Fordtram JS. Subepithelial collagen table thickness in colon specimes from patients with microscopic colitis and collagenous colitis. Gastroenterology 1992; 103: 1970-1976.

40. Miquel Plaza J, López San Román A; del Pozo D, Pena E, Bermejo F, Baleriola I, et al. Evolution and treatment response in microscopic colitis. Gastroenterol Hepatol 2001; 24 (9): 433-9. 